\title{
Macroscopic stress from dynamic, rotating granular media
}

\author{
S. Luding \\ Multi Scale Mechanics, TS, CTW, UTwente, \\ POBox 217, 7500 AE Enschede, Netherlands; \\ e-mail: s.luding@utwente.nl
}

\begin{abstract}
In this brief study, the stress is averaged over a two-dimensional rigid particle (disk) that is in contact with other objects via localized (point) contacts. In contrast to previous studies, the rotation dynamics of the particles is also taken into account here The stress contains four terms, (i) the static stress due to forces, (ii) the dynamic stress due to translational velocity fluctuations, (iii) the dynamic stress due to rotational velocity fluctuations, and (iv) the stress due to changes of angular velocities due to torques.
\end{abstract}

Keywords: dynamic stress, rotations, averaging, fluctuations, non-equilibrium

PACS: $45.70,47.50+\mathrm{d}, 51.10 .+\mathrm{y}$

\section{INTRODUCTION}

One of todays great challenges in material science and physics is the macroscopic description of the material behavior of granular media which are inhomogeneous, nonlinear, and disordered on a "microscopic" scale [1, 2, 3, 4]. This is due to the intrinsically inhomogeneous stress distribution in granular assemblies and the corresponding stress-networks involving large fluctuations of contact forces. Furthermore, the material is nonlinear due to the nature of the contact forces: If a contact opens, the interaction force vanishes or, with other words, no tensile but only compressive forces are possible in the absence of cohesion. This nonlinearity allows for a reorganization of the contact-network due to deformation.

While there is quite broad consensus on how stress should be averaged $[5,6,7,8,9,10,11,12,13,14,15,16$, $17,18,19,20]$, the averaging of strain is still a subject of discussion, see Ref. [21] and references therein. In many traditional studies the stress is averaged under the assumption of static equilibrium $[8,9,22,11,13,23]$. However, in this study we allow for a dynamic stress, where the particles are not in equilibrium and thus moving and rotating. The stress is first averaged over single particles, for which several simplifications can be applied. Finally, the resulting stress tensor is interpreted term by term.

\section{STRESS AVERAGING}

In a first step, the usual volume average of stress is replaced by a sum over particle averaged internal stresses

$$
\underline{\underline{\bar{\sigma}}}=\frac{1}{V} \int_{V} \underline{\underline{\sigma}} d V^{\prime}=\frac{1}{V} \sum_{p} V^{p} \underline{\underline{\underline{\sigma}}}^{p}:=\frac{1}{V} \sum_{p} \int_{V_{p}} \underline{\underline{\sigma}} d V^{\prime}
$$

where $p$ denotes the particle with volume $V^{p}$ and the sum runs over all particles within the volume $V .{ }^{1}$ The integral on the right hand side is carried out only over a single particle (where the stress in the free volume between particles is assumed to vanish and thus neglected). This operation will allow us to deal with particle averaged stresses $\underline{\underline{\sigma}}^{p}$ instead of volume averages. For the sake of simplicity we skip the stress-superscript $p$ in the following.

\footnotetext{
${ }^{1}$ Note that the weight factor $V^{p}$ in the sum cancels the prefactor $1 / V^{p}$ in the implicit definition of the particle stress, where the particle stress is averaged over points strictly inside the particle only. This is matter of choice and $V^{p}$ could be replaced by $V_{c}^{p}$ at all instances, which would define the particle stress in its Voronoi cell, with $\sum_{p} V_{c}^{p}=V$. We do not apply this latter definition - which would not change the averaged stress anyway - since the volumes $V_{c}^{p}$ are a-priori unknown and in general different per particle in a disordered packing.

Assume a single particle $p$, with $V^{p}=\pi a^{2}$, and constant stress $\sigma_{0}$ in its inside. When it occupies, e.g., a square cell with volume $V=4 a^{2}$, the averaged internal particle stress is $\sigma^{p}=\sigma_{0}$, while the average stress over the volume is $\bar{\sigma}=v \sigma_{0}$, with volume fraction $v=V^{p} / V$
} 
In order to proceed, we first introduce the identity for the transposed ${ }^{2}$ stress

$$
\underline{\underline{\sigma}}^{T}=(\operatorname{grad} \vec{x}) \cdot \underline{\underline{\sigma}}^{T}=\operatorname{div}(\vec{x} \otimes \underline{\underline{\sigma}})-\vec{x} \otimes \operatorname{div} \underline{\underline{\sigma}},
$$

where $\operatorname{grad}$ and div are the usual vector operator derivatives with respect to $\vec{x}, \operatorname{grad} \vec{x}$ is the unit tensor, and $\otimes$ is the dyadic product of vectos or tensors. Combinig Eqs. (1) and (2) yields

$$
\underline{\underline{\bar{\sigma}}}^{T}=\left(\underline{\underline{\bar{\sigma}}}^{p}\right)^{T}=\frac{1}{V^{p}} \int_{V^{p}} \underline{\underline{\sigma}}^{T} d V^{\prime}=\frac{1}{V^{p}} \int_{V^{p}}[\operatorname{div}(\vec{x} \otimes \underline{\underline{\sigma}})-\vec{x} \otimes \operatorname{div} \underline{\underline{\sigma}}] d V^{\prime}
$$

The law of momentum balance in Eulerian reference frame, for the volume occupied by particle $p$ at time $t$, reads

$$
\rho \ddot{\vec{x}}+\rho \vec{v} \cdot \operatorname{grad} \vec{v}=\operatorname{div} \underline{\underline{\sigma}}+\rho \vec{b}
$$

where the double-dots are partial derivatives with respect to time and $\vec{b}$ is an external acceleration, e.g. gravity. Inserting Eq. (4) in Eq. (3) (and restricting to one particle) leads to

$$
\underline{\underline{\sigma}}^{T}=\frac{1}{V^{p}}[\underbrace{\int_{\partial V p} \vec{x} \otimes \underline{\underline{\sigma}} \cdot d \vec{A}}_{\underline{\underline{\sigma}}_{S}^{T}}-\underbrace{\int_{V^{p}} \vec{x} \otimes \rho(\ddot{\vec{x}}-\vec{b}) d V^{\prime}}_{-\underline{\underline{\sigma}}_{v}^{T}}-\underbrace{\int_{V^{p}} \vec{x} \otimes \rho(\vec{v} \cdot \operatorname{grad} \vec{v}) d V^{\prime}}_{-\underline{\underline{\sigma}}_{d}^{T}}],
$$

where the first term comes from Gauss' theorem that allows to translate a volume integral into a surface integral. The term that involves the constant acceleration is merged into the second term. The three stress contributions will be addressed seperately below.

\section{The surface integral of Eq. (5)}

Using the Cauchy theorem $\vec{t}=\underline{\sigma} \cdot \vec{n}$ and the definition $d \vec{A}=\vec{n} d A$, with the normal $\vec{n}$ to the boundary $\partial V^{p}$ of particle $p$, allows to transform the first part of Eq. (5) into a sum [23]:

$$
\underline{\underline{\sigma}}_{s}^{T}=\frac{1}{V^{p}} \int_{\partial V^{p}}(\vec{x} \otimes \vec{t}) d A=\frac{1}{V^{p}} \sum_{c} \vec{x}^{c} \otimes \vec{f}^{c}
$$

after replacing the surface stresses active at the contacts by the corresponding forces $\vec{f}^{c}$. Note that here very small contact areas are assumed, so that the integral can be transformed into the sum in Eq. (6), which represents the transition from the Eulerian (continuum) to the Lagrangian (discrete) picture, respectively.

Introducing the branch vector $\vec{l}^{p c}$ by the vector addition $\vec{x}^{c}=\vec{x}^{p}+\vec{l}^{p c}$, as shown in Fig. 1, leads to

$$
\underline{\underline{\sigma}}_{s}^{T}=\frac{1}{V^{p}}\left[\vec{x}^{p} \otimes \sum_{c} \vec{f}^{c}+\sum_{c} \vec{l}^{p c} \otimes \vec{f}^{c}\right] .
$$

With Newtons law for the motion of particle $p$ with mass $m$,

$$
m \ddot{\vec{x}}^{p}=\sum_{c} \vec{f}^{c}+m \vec{b}
$$

one finally gets

$$
\underline{\bar{\sigma}}_{s}^{T}=\frac{1}{V^{p}}\left[m \vec{x}^{p} \otimes\left(\ddot{\vec{x}}^{p}-\vec{b}\right)+\sum_{c} \vec{l}^{p c} \otimes \vec{f}^{c}\right]
$$

for the first integral in Eq. (5).

\footnotetext{
2 The transposed stress is introduced on the left hand side of Eq. (2) just to keep the $T$-symbol out of the integrals for convenience. The stress in Eq. (1) is then just the transposed of the transposed stress. For symmetric stresses, the $T$ could be dropped completely, however, our result is non-symmetric in general, dynamic situations.
} 


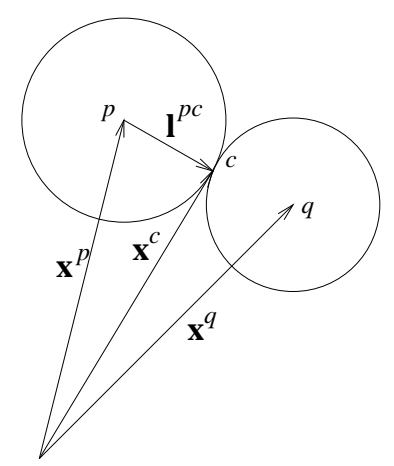

FIGURE 1. Schematic plot of two particles $p$ and $q$ with their common contact $c$.

\section{The volume integral of Eq. (5)}

The volume integral

$$
\underline{\underline{\sigma}}_{v}^{T}=-\frac{1}{V^{p}} \int_{V^{p}}(\vec{x} \otimes \rho \ddot{\vec{x}}-\vec{x} \otimes \rho \vec{b}) d V^{\prime}
$$

contains those terms acting on all material points of particle $p$. Therefore, one has to introduce a vector $\vec{l}$ which points from the center of mass of the particle to the material points inside so that $\vec{x}=\vec{x} p+\vec{l}$, see Fig. 2 .

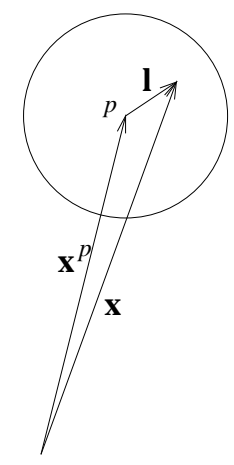

FIGURE 2. Schematic plot of a particle $p$ and its material point $\vec{x}$.

This leads to

$$
{\underline{\underline{\bar{\sigma}^{2}}}}_{v}^{T}=-\frac{1}{V^{p}} \int_{V^{p}}\left(\vec{x}^{p}+\vec{l}\right) \otimes \rho\left(\ddot{\vec{x}}^{p}+\ddot{\vec{l}}-\vec{b}\right) d V^{\prime}
$$

where the vectors $\vec{x}^{p}$ and $\vec{b}$ are constant, so that one can draw them out of the integral. The integral $\int_{V^{p}} \rho d V^{\prime}$ is the mass $m$ of the particle as implied in the following. In separate terms the stress reads

$$
\begin{aligned}
\underline{\underline{\sigma}}_{v}^{T}=-\left(1 / V^{p}\right)[ & +m \vec{x}^{p} \otimes \ddot{\vec{x}}^{p} \\
& +\vec{x}^{p} \otimes \int_{V^{p}} \rho \ddot{\vec{l}} d V^{\prime} \\
& -m \vec{x}^{p} \otimes \vec{b} \\
& +\left(\int_{V^{p}} \rho \vec{l} d V^{\prime}\right) \otimes \ddot{\vec{x}}^{p} \\
& +\int_{V^{p}} \rho \vec{l} \otimes \ddot{\vec{l}} d V^{\prime} \\
& \left.-\left(\int_{V^{p}} \rho \vec{l} d V^{\prime}\right) \otimes \vec{b}\right]
\end{aligned}
$$


The fourth term, Eq. (15), and the sixth term, Eq. (17), vanish due to the fact that $\int_{V^{p}} \rho \vec{l} d V^{\prime}$ is the definition of the center of mass and $\vec{l}$ is defined relative to the center of mass. For the rotational motion of a rigid body with angular velocity $\omega$ around its center of mass one has

$$
\begin{aligned}
\dot{\vec{l}} & =\vec{\omega} \times \vec{l}, \text { and } \\
\overrightarrow{\vec{l}} & =\dot{\vec{\omega}} \times \vec{l}+\vec{\omega} \times \overrightarrow{\vec{l}} \\
& =\dot{\vec{\omega}} \times \vec{l}+\vec{\omega} \times(\vec{\omega} \times \vec{l})
\end{aligned}
$$

so that also the second term, Eq. (13), equals zero because both $\vec{\omega}$ and $\dot{\vec{\omega}}$ are constant over the rigid particle and thus can be drawn out of the integral. Finally, using $\vec{\omega} \times(\vec{\omega} \times \vec{l})=\vec{\omega}(\vec{\omega} \cdot \vec{l})-\vec{l}\left(\omega^{2}\right)=-\vec{l}\left(\omega^{2}\right)$, since $\vec{\omega}$ and $\vec{l}$ are perpendicular in 2D disks rotating around their axis of rotational symmetry, one gets

$$
-\frac{1}{V^{p}}\left[m \vec{x}^{p} \otimes\left(\ddot{x}^{p}-\vec{b}\right)+\int_{V^{p}} \rho \vec{l} \otimes\left(\dot{\vec{\omega}} \times \vec{l}-\omega^{2} \vec{l}\right) d V^{\prime}\right] .
$$

Using the identity $\vec{l} \otimes(\dot{\vec{\omega}} \times \vec{l})=-(\vec{l} \otimes \vec{l}) \times \dot{\vec{\omega}}$ and drawing the constants out of the integrals, yields

$$
\underline{\underline{\sigma}}_{v}^{T}=-\frac{1}{V^{p}}\left[m \vec{x}^{p} \otimes\left(\ddot{\vec{x}}^{p}-\vec{b}\right)+\underline{\underline{\theta}} \times \dot{\vec{\omega}}+\omega^{2} \underline{\underline{\theta}}\right],
$$

after introducing the symmetric tensor $\underline{\underline{\theta}}:=-\int_{V^{p}} \rho \vec{l} \otimes \vec{l}$.

\section{The dynamic stress in Eq. (5)}

The integral

$$
\underline{\underline{\sigma}}_{d}^{T}=-\frac{1}{V^{p}} \int_{V^{p}}(\vec{x} \otimes \rho \vec{v} \cdot \operatorname{grad} \vec{v}) d V^{\prime}
$$

can be simplified by transforming the components of the term

$$
-x_{\alpha} v_{\gamma} v_{\beta, \gamma}=-\left(x_{\alpha} v_{\gamma} v_{\beta}\right)_{, \gamma}+x_{\alpha, \gamma} v_{\gamma} v_{\beta}+x_{\alpha} v_{\gamma, \gamma} v_{\beta},
$$

where the,$\gamma$ replaces the gradient. The last term on the r.h.s. vanishes due to the incompressibility of the particles $v_{\gamma, \gamma}=0$. The first term leads to a surface integral using the Cauchy theorem, however, it vanishes since $\vec{n} \cdot \dot{\vec{l}}=0$, and the surface integral over terms $\vec{v}^{p} \cdot \vec{n}$ also vanishes due to the symmetric particle shape. The second integral survives and, after replacing grad $\vec{x}$ by the unit tensor, has to be treated in a way similar to the volume integral in the previous subsection. Therefore, we replace the vector $\vec{v}$ by $\dot{\vec{x}}=\dot{\vec{x}}^{p}+\dot{\vec{l}}$, so that

$$
\underline{\underline{\sigma}}_{d}^{T}=\frac{1}{V^{p}} \int_{V^{p}}\left(\rho\left(\dot{\vec{x}}^{p}+\dot{\vec{l}}\right) \otimes\left(\dot{\vec{x}}^{p}+\dot{\vec{l}}\right)\right) d V^{\prime}
$$

Since the mixed terms contain $\dot{\vec{x}}^{p} \otimes \dot{\vec{l}}$ and thus vanish due to the definition of $\vec{l}$. The dyadic velocity tensor $\rho \dot{\vec{x}}^{p} \otimes \dot{\vec{x}}^{p}$ can be easily integrated so that the remaining integral contains

$$
\dot{\vec{l}} \otimes \dot{\vec{l}}=(\vec{\omega} \times \vec{l}) \otimes(\vec{\omega} \times \vec{l})=\omega^{2}\left(l^{2} \underline{\underline{I}}-\vec{l} \otimes \vec{l}\right) .
$$

The integral over the first term leads to $m a^{2} \omega^{2} \underline{\underline{I}} / 2$, with the particle radius $a$, and the second term is again $\underline{\underline{\theta}}$, so that the dynamic stress is

$$
\underline{\underline{\sigma}}_{d}^{T}=\rho\left[\vec{v}^{p} \otimes \vec{v}^{p}+\frac{1}{2} a^{2} \omega^{2} \underline{\underline{I}}\right]+\frac{1}{V^{p}} \omega^{2} \underline{\underline{\theta}} .
$$




\section{The combined particle stress}

Inserting Eqs. (9), (20), and (25) in Eq. (5), finally leads to

$$
\left(\underline{\underline{\bar{\sigma}}}^{p}\right)^{T}=\underline{\underline{\bar{\sigma}}}^{T}=\frac{1}{V^{p}}\left[\sum_{c} \vec{l}^{p c} \otimes \vec{f}^{c}+m \vec{v}^{p} \otimes \vec{v}^{p}-\underline{\underline{\theta}} \times \dot{\vec{\omega}}+\underline{\underline{\theta}}_{0} \omega^{2}\right]
$$

with the moment of inertia tensor for rotation around the disk center $\underline{\underline{\theta}}_{0}=(\mathrm{m} / 2) a^{2} \underline{\underline{I}}$.

\section{The many-particle macroscopic stress}

Inserting the internal averaged particle stress from Eq. (26) into Eq. (1) finally leads to the macroscopic averaged many particle stress:

$$
\underline{\underline{\sigma}}=\frac{1}{V} \sum_{p} V^{p} \underline{\underline{\sigma}}^{p}=\frac{1}{V} \sum_{c \in V} \vec{f}^{c} \otimes \vec{l}^{p c}+\frac{1}{V} \sum_{p}\left[m \vec{u}^{p} \otimes \vec{u}^{p}-\dot{\vec{\omega}}^{p} \times \underline{\underline{\theta}}+\underline{\underline{\theta}}_{0}\left(\omega^{p}\right)^{2}\right]
$$

where $\vec{v}^{p}=\vec{v}_{c m}+\vec{u}^{p}$ in Eq. (26) contained still both the average center of mass (cm) velocity and the fluctuation velocities $u$. The constant, moving reference frame "stress" $\underline{\underline{\sigma}}_{c m}=\frac{M}{V} \vec{v}_{c m} \otimes \vec{v}_{c m}$ is divergence-free in Eq. (4) and therefore can be disregarded in order to gain objectivity and frame independence of the stress.

\section{DISCUSSION}

The first stress term in Eq. (26) is the well-known, static contribution to the stress tensor and the second term is the dynamic (kinetic) contribution due to the fluctuating particle motion with respect to the Eulerian reference frame ( $\rightarrow$ for details see the kinetic theory of gases), i.e. a kinetic energy density. The third, asymmetric term is related to the change of angular velocity and, thus, couples the non-equilibrium translational degrees of freedom to the non-equilibrium rotational motion. Finally, the last term is the rotational energy density, paralleling the dynamic, translational contribution to the stress tensor. Note that our formulation is not identical with the improved stress formulation as recently proposed by Goldhirsch [20], which in principle allows for non-symmetric stress also in the case of static equilibrium.

Comparison of the different analytical stress predictions among each other, with particle simulation results and with experiments is the next step to be done in order to gain improved insight about the effects of dynamics on the stress in granular media.

\section{OUTLOOK}

After having performed the above calculations with various assumptions (rigid disk in a two dimensional system) one might wish to compute the stress tensor for the more general case of three-dimensional, possibly non-spherical, objects with internal degrees of freedom like vibrational modes.

While the generalization to 3D spheres sees straightforward, the consequences of a non-sperical geometry and some non-rigidity might complicate the integrals too much to allow a comparatively straight approach.

\section{ACKNOWLEDGMENTS}

Helpful discussions with M. Lätzel, N. P. Kruyt, S. Diebels, W. Volk, W. Ehlers, and H. J. Herrmann are gratefully acknowledged. This work was made possible by the financial support of the Deutsche Forschungsgemeinschaft (DFG) and the Stichting voor Fundamenteel Onderzoek der Materie (FOM), financially supported by the Nederlandse Organisatie voor Wetenschappelijk Onderzoek (NWO). 


\section{REFERENCES}

1. H. J. Herrmann, J.-P. Hovi, and S. Luding, editors, Physics of dry granular media - NATO ASI Series E 350, Kluwer Academic Publishers, Dordrecht, 1998.

2. P. A. Vermeer, S. Diebels, W. Ehlers, H. J. Herrmann, S. Luding, and E. Ramm, editors, Continuous and Discontinuous Modelling of Cohesive Frictional Materials, Springer, Berlin, 2001, lecture Notes in Physics 568.

3. Y. Kishino, editor, Powders \& Grains 2001, Balkema, Rotterdam, 2001.

4. M. Nakagawa, and S. Luding, editors, Powders and Grains 2009, American Institute of Physics (AIP), Golden, Colorado, USA, 2009, confernce Proceedings \#1145, ISBN 978-0-7354-0682-7.

5. A. Drescher, and G. de Josselin de Jong, J. Mech. Phys. Solids 20, 337-351 (1972).

6. L. Rothenburg, and A. P. S. Selvadurai, "A micromechanical definition of the Cauchy stress tensor for particulate media," in Mechanics of Structured Media, edited by A. P. S. Selvadurai, Elsevier, Amsterdam, 1981, pp. 469-486.

7. S. B. Savage, and D. J. Jeffrey, J. Fluid. Mech. 110, 255 (1981).

8. P. A. Cundall, A. Drescher, and O. D. L. Strack, "Numerical experiments on granular assemblies; Measurements and observations," in IUTAM Conference on Deformation and Failure of Granular Materials, Delft, 1982, pp. 355-370.

9. J. D. Goddard, "Microstructural Origins of Continuum Stress Fields - a Brief History and Some unresolved Issues," in Recent Developments in Structered Continua. Pitman Research Notes in Mathematics No. 143, edited by D. DeKee, and P. N. Kaloni, Longman, J. Wiley, New York, 1986, p. 179.

10. R. J. Bathurst, and L. Rothenburg, J. Appl. Mech. 55, 17-23 (1988).

11. N. P. Kruyt, and L. Rothenburg, ASME Journal of Applied Mechanics 118, 706-711 (1996).

12. J. J. Moreau, "Numerical Investigation of Shear Zones in Granular Materials," in Friction, Arching and Contact Dynamics, edited by D. E. Wolf, and P. Grassberger, World Scientific, Singapore, 1997.

13. C.-L. Liao, T.-P. Chang, D.-H. Young, and C. S. Chang, Int. J. Solids \& Structures 34, 4087-4100 (1997).

14. I. Goldhirsch, and C. Goldenberg, Eur. Phys. J. E 9, 245-251 (2002).

15. C. Goldenberg, and I. Goldhirsch, Phys. Rev. Lett. 89, 084302 (2002).

16. C. Goldenberg, and I. Goldhirsch, Granular Matter 6, 87-96 (2004).

17. L. Staron, F. Radjai, and J.-P. Vilotte, Eur. Phys. J. E 18, 311-320 (2005).

18. C. Goldenberg, and I. Goldhirsch, Nature 435, 188-191 (2005).

19. C. Goldenberg, A. P. F. Atman, P. Claudin, G. Combe, and I. Goldhirsch, Scale separation in granular packings: stress plateaus and fluctuations (2005), submitted to Physical Review Letters; e-print: cond-mat/0511610.

20. I. Goldhirsch, Granular Matter 12 (2010), submitted.

21. O. Duran, N. P. Kruyt, and S. Luding, Int. J. of Solids and Structures 47, 251-260 (2010).

22. C. S. Chang, "Micromechanical modelling of constitutive relations for granular media," in Micromechanics of granular media, Elsevier, Amsterdam, 1988.

23. M. Lätzel, S. Luding, and H. J. Herrmann, Granular Matter 2, 123-135 (2000), e-print cond-mat/0003180. 